\title{
Tendência e distribuição da taxa de mortalidade por homicídios segundo porte populacional dos municípios do Brasil, 2000 e 2015
}

\author{
Trend and distribution of the homicide mortality rate \\ in accordance with the size of the population of Brazilian \\ municipalities - 2000 and 2015
}

\begin{abstract}
Adauto Martins Soares Filho (https://orcid.org/0000-0002-0917-7473) ${ }^{1}$
Elisabeth Carmen Duarte (https://orcid.org/0000-0001-9148-5063) ${ }^{2}$

Edgar Merchan-Hamann (https://orcid.org/0000-0001-6775-9466) ${ }^{3}$
\end{abstract}

${ }^{1}$ Secretaria de Vigilância em Saúde, Ministério da Saúde. Esplanada dos Ministérios Bloco G/Térreo, Asa Sul. 70058-900 Brasília DF Brasil.

afilho_2006@hotmail.com ${ }^{2}$ Faculdade de Medicina, Universidade de Brasília (UnB). Brasília DF Brasil. ${ }^{3}$ Faculdade de Ciências da Saúde, UnB. Brasília DF Brasil.

\begin{abstract}
The scope of this study is to analyze the trends and distribution of homicide mortality rates (HMR) according to the population size of Brazilian municipalities between 2000 and 2015. It is an ecological study of deaths recorded in the Mortality Information System, with HMR standardized by the direct method and 95\% confidence in terval. HMR in Brazil grew 6\% (to 29.1/100,000) in the period, with an increase in small municipalities (83\%; 12.7 to 23.2/100,000) and mediumsized cities (52\%; 19.7\% to 30.1/100,000); which is true for both sexes, different ages, regions and firearm-related events. HMR decreased in major cities (19\%; 40.6\% to 32.9/100,000) and the Southeast region (55\%; $45.6 \%$ to 20.6/100,000). The relative risk $(R R)$ of small and medium-sized cities in relation to large cities is already greater than or close to 1 among women (RR 0.99; 1.03), people aged 60 years or older $(R R 1.43 ; 1.36)$ and homicides by other means (RR 1.16; 1.18). The cities of São Paulo and Rio de Janeiro contributed the most to the reduction of HMR, especially in large cities (-37.6 and -22.3 homicides/100,000 inhabitants). Small and medium-sized municipalities have consistent trends of an increase in HMR even considering population subgroups.

Key words Homicide, Mortality, Space-time clustering, Ecological studies
\end{abstract}

Resumo $O$ estudo tem como objetivo analisar tendências e distribuição das taxas de mortalidade por homicídios (TMH) segundo porte populacional dos municípios brasileiros entre 2000 e 2015. Trata-se de estudo ecológico dos óbitos do Sistema de Informações sobre Mortalidade, com TMH padronizada pelo método direto e intervalo de 95\% de confiança. A TMH no Brasil cresceu 6\% (para 29,1/100 mil) no período, com aumento em municípios de pequeno (83\%; 12,7 para 23,2/100 mil) e médio porte (52\%; 19,7 para 30,1/100 mil); o que se verifica para ambos os sexos, diferentes idades, regiões e em eventos por arma de fogo. A TMH decresceu em municipios de grande porte metropolitanos (19\%; 40,6 para 32,9/100 mil) e da região Sudeste (55\%; 45,6 para 20,6/100 mil). $O$ risco relativo- $R R$ de cidades pequenas e médias em relação a grandes já é maior ou próximo de 1 em mulheres (RR 0,99; 1,03), pessoas com 60 ou mais anos $(R R 1,43 ; 1,36)$ e homicídios por outros meios (RR 1,16; 1,18). As cidades de São Paulo e Rio de Janeiro foram as que mais contribuíram para a redução das $T M H$, em especial nas cidades grandes (-37,6 e -22,3 homicídios/100 mil hab.). Municípios de porte pequeno e médio apresentam tendências consistentes de incremento de TMH mesmo considerando subgrupos populacionais.

Palavras-chave Homicídio, Mortalidade, Conglomerados espaço-temporais, Estudos ecológicos 


\section{Introdução}

Estima-se que 408.583 pessoas foram vítimas de homicídio no mundo, em 2015. Esses eventos representam uma taxa de mortalidade por homicídio (TMH) de 5,5 por 100 mil habitantes ${ }^{1}$. As mortes por essas causas na América-Latina e Caribe representam 34\% (139.200) do total de casos no mundo, e uma TMH de 23,6 por 100 mil habitantes: o maior risco global. El Salvador (58,3 por $100 \mathrm{mil} \mathrm{hab})$, Honduras $(48,4$ por $100 \mathrm{mil}$ hab), Venezuela (43,8 por 100 mil hab) e Guatemala (39,5 por 100 mil hab) são os países dessa região que exibem as maiores $\mathrm{TMH}^{1}$.

O Brasil, com 61.237 mortes por homicídio em 2015, apresentou TMH de 27,8 por 100 mil hab ${ }^{1}$. Predominam os eventos ocorridos por agressão por arma de fogo, em via pública, próxima à residência, nas áreas mais pobres e periferias das cidades brasileiras, principalmente em estados da região Nordeste. O tráfico (drogas e armas) e as desigualdades sociais são fatores contextuais que favorecem a ocorrência de homicídios também frequentemente discutidos por alguns autores ${ }^{2,3}$.

Homens adultos jovens, negros, de baixo nível socioeconômico e baixa escolaridade e consumo de álcool e drogas são os fatores de risco individuais mais claramente associados à maior risco de morte por homicídio ${ }^{4-6}$. Mulheres assassinadas, embora em menor magnitude, também apresentam perfil semelhante ${ }^{7-9}$. Interessante notar que o padrão das vítimas e do autor da violência não difere de forma importante, ambos adultos jovens, negros e de baixa escolaridade ${ }^{10-13}$.

$\mathrm{Na}$ última década, o homicídio no Brasil distingue-se por apresentar redução no Sudeste, principalmente nos estados de São Paulo e Rio de Janeiro. Essa queda também é verificada em ambos os sexos e nas regiões metropolitanas das capitais desses estados ${ }^{14-16}$.

Embora os perfis das vítimas e dos agressores tenham sido amplamente estudados, pouco se conhece sobre as tendências recentes das TMH no Brasil segundo características contextuais dos municípios. Aprofundar neste entendimento auxilia na identificação dos municípios mais vulneráveis e no levantamento de hipóteses explicativas para fenômeno tão complexo como é a etiologia da violência no Brasil. Assim, este estudo tem por objetivo analisar tendências e distribuição das TMH segundo porte populacional dos municípios brasileiros, entre 2000 e 2015.

\section{Métodos}

Estudo ecológico, que avalia a tendência das TMH entre 2000 e 2015, segundo o porte populacional dos municípios brasileiros. A comparação das taxas entre os anos oportuniza a identificação de possíveis mudanças no padrão de mortalidade. Os dados de óbitos, por local de residência, foram os últimos registros disponíveis do Sistema de Informações sobre Mortalidade (SIM) do Ministério da Saúde ao início do estudo ${ }^{17}$. A fonte de dados das populações foi o Instituto Brasileiro de Geografia e Estatística (IBGE), via Departamento de informática do SUS (Datasus) ${ }^{18}$.

As mortes por homicídio foram identificadas pelos códigos da Classificação Internacional de Doenças (CID 10) ${ }^{19}$ e incluíram: agressões (X85-Y09), eventos de intenção indeterminada por arma de fogo (Y22 a Y24) e intervenções legais $(\mathrm{Y} 35)^{20}$. As TMH foram calculadas por 100 mil habitantes, padronizadas por idade pelo método direto. A população padrão usada foi a identificada pelo censo de $2010^{18}$. As TMH padronizadas por idade foram desagregadas por sexo e idade, para os anos de 2000 e 2015.

A principal variável explicativa de interesse foi porte populacional do município de residência da vítima. Para tanto, os municípios foram agrupados de acordo com o número de habitantes em 2000 e 2015 e categorizados em três extratos de porte populacional: pequeno porte $(<$ 50 mil habitantes), médio porte $(50 \mathrm{a}<100 \mathrm{mil}$ habitantes) e grande porte (100 mil ou mais habitantes).

Outras variáveis analisadas foram: tipo de arma (arma de fogo, outros meios), sexo (masculino, feminino) e idade (0-14, 15-29, 30-59, 60 ou + anos de idade) da vítima, e região metropolitana (sim, não) e região (Norte, Nordeste, Sudeste, Sul, Centro-oeste) do município de residência da vítima.

As TMH foram comparadas usando seus respectivos intervalos de 95\% de confiança. A hipótese de não diferença $\left(\mathrm{H}_{0}\right)$ das TMH foi refutada quando os intervalos de confiança das taxas não se sobrepunham, i.e., assume-se a hipótese alternativa $\left(\mathrm{H}_{1}\right)$ que as taxas eram significativamente diferentes, nas comparações de 2000 e 2015, indicando tendência crescente ou decrescente no período.

Além disso, a variação da TMH por 100 mil habitantes foi analisada corrigindo o impacto do crescimento populacional, segundo porte populacional dos municípios e microrregiões das unidades federadas do país. Essa variação corrigida (VC) é definida por: 
$\mathrm{VC}=\frac{\left(\text { óbito }^{\mathrm{f}}-\left(\left(\text { populaçãof população }^{\mathrm{i}}\right)^{\star} \text { óbito }^{\mathrm{i}}\right)\right)}{\text { população }^{\mathrm{f} \star} 100.000}$

Onde $\mathrm{f}$ (final) é o dado referente ao último ano do período, e i (inicial) é o dado do primeiro ano do período.

A análise foi feita com auxílio do software "Programa para Análises Epidemiológicas de Dados Tabulados" (Epidata), versão 3.1, da Organização Panamericana de Saúde (OPAS)/Xunta de Galícia.

O estudo usou exclusivamente dados secundários não nominal, em conformidade com o Decreto $\mathrm{n}^{\circ} 7.724^{21}, 16$ de maio de 2012 que dispõe sobre o acesso a informações e a Resolução $\mathrm{n}^{\circ} 510^{22}$, de 7 de abril de 2016 que dispõe sobre as normas aplicáveis a pesquisas.

\section{Resultados}

Nos anos de 2000 e 2015, o Brasil apresentou, respectivamente, 46.698 e 59.083 mortes e taxas de mortalidade por homicídios (TMH) de 27,4 (IC95\% 27,2; 27,7) e 29,1 (IC95\% 28,9; 29,4) por 100 mil habitantes. Em ambos os anos da análise, as maiores TMH foram observadas em municípios de grande porte, de áreas metropolitanas, como consequência principalmente do uso de arma de fogo, cujas vítimas eram principalmente jovens (15 a 29 anos) do sexo masculino (Tabela 1 ). As regiões que se destacaram com maiores TMH no ano de 2000 foram a Centro Oeste, Nordeste e Sudeste (estas duas últimas principalmente para os municípios de grande porte), e no ano de 2015 foram as regiões Centro Oeste, Nordeste e Norte.

A TMH apresentou crescimento, entre $2000 \mathrm{e}$ 2015, de $83 \%$ nos municípios de pequeno porte e de $52 \%$ nos municípios de médio porte. Já nos municípios de grande porte, a TMH apresentou decréscimo de 15\% nos mesmo período (Tabela 1).

Essas tendências de crescimento das TMH nos municípios pequenos e médios, e decréscimo nos municípios de grande porte foram também observadas quando as TMH são analisadas separadamente segundo tipo de arma (arma de fogo ou outros meios), segundo sexo (homens e mulheres) e segundo diferentes grupos de idade (Tabela 1). Por exemplo, observou-se crescimento acentuado da TMH em municípios de pequeno porte entre os homens $(88 \%)$ e entre as pessoas de 15 a 29 anos de idade (128\%), e decréscimo da TMH nestes mesmos grupos populacionais em municípios de grande porte (Tabela 1).
A TMH também cresceu nos municípios de pequeno e médio porte independente destes pertencerem ou não às regiões metropolitanas. Por outro lado, a TMH decresceu nos municípios de grande porte apenas quando eram metropolitanos (-19\%; 40,6 para 32,9/100 mil), e cresceu discretamente em municípios de grande porte não metropolitanos $(20 \% ; 24,1$ para 29/100 mil) (Tabela 1).

Com respeito às regiões, o padrão verificado anteriormente se repete na região Sudeste: a TMH cresceu significativamente nos pequenos municípios (42\%; 10,6 para 15,1/100 mil), ficou estável nos municípios médios (diferença não significativa) e decresceu nos grandes municípios (-55\%; 45,6 para 20,6/100 mil) (Tabela 1). Para as demais regiões, no entanto, a TMH apresentou crescimento significativo e independente do porte dos municípios (Tabela 1).

A razão de TMH (risco relativo $=\mathrm{RR})$ comparando os municípios de pequeno porte com os de grande porte aponta para maior homogeneidade em $2015(\mathrm{RR}=0,72)$, quando comparado ao ano de $2000(R R=0,34)$ (Tabela 2). Isso se repete para quase a totalidade das variáveis estudadas, entre 2000 e 2015. Por exemplo, observaramse aumentos dos RR resultado da razão das TMH em municípios pequenos e médios comparados aos grandes entre 2000 e 2015 para os homens $(\mathrm{RR}=0,31$ para 0,68$)$, grupo etário de 15 a 29 anos $(\mathrm{RR}=0,22$ para 0,55$)$, área metropolitana $(\mathrm{RR}=0,33$ para 0,81$)$ e região Sudeste $(\mathrm{RR}=0,23$ para 0,73$)$. Na região Sul, no entanto, a razão de TMH (RR) permaneceu estável entre 2000 e 2015 para a comparação dos municípios menores com os de grande porte (diferença não significativa), e apresentou redução para a comparação dos municípios médios $(-13 \% ; R R=0,77$ para 0,67$)$ em relação aos de grande porte (Tabela 2).

Entre 2000 e 2015, as TMH apresentaram redução nas três categorias de porte populacional de município dos estados de São Paulo e Rio de Janeiro. Maiores reduções dessas taxas nesses dois estados foram observadas nos municípios de grande porte, como segue: $-37,6$ e -22,3 homicídios por 100 mil habitantes, respectivamente (Figura 1). Pernambuco, Espírito Santo e Rondônia apresentaram redução das TMH em municípios grandes e médios, sobretudo nos primeiros, a saber: - 25,2, -18,9 e -12,7 homicídios/100 mil hab, respectivamente. Os estados de Mato Grosso do Sul, Distrito Federal, Mato Grosso, Roraima, Amapá e Acre apresentaram redução das TMH nos municípios de grande porte. Os demais estados brasileiros exibiram crescimento das TMH 


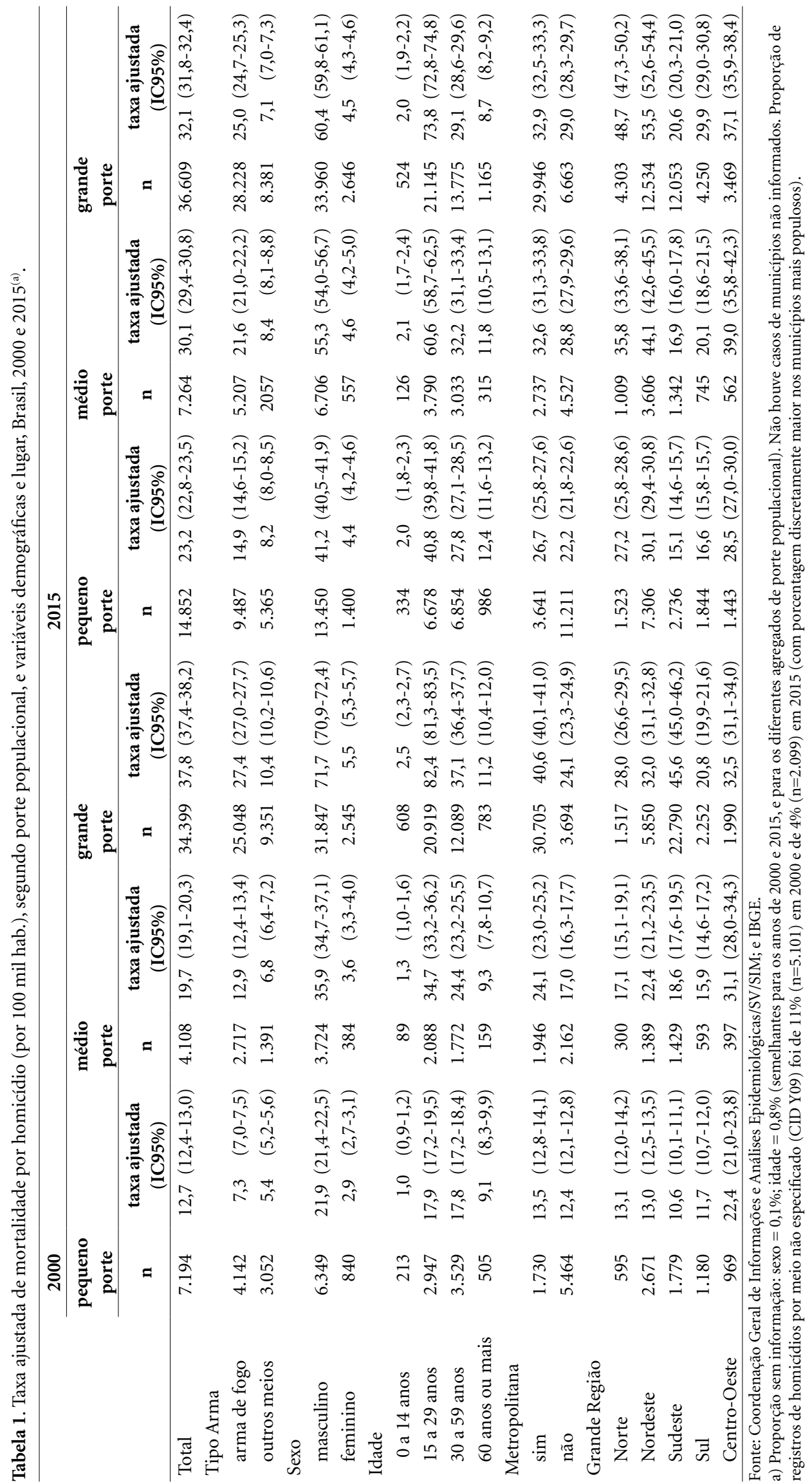




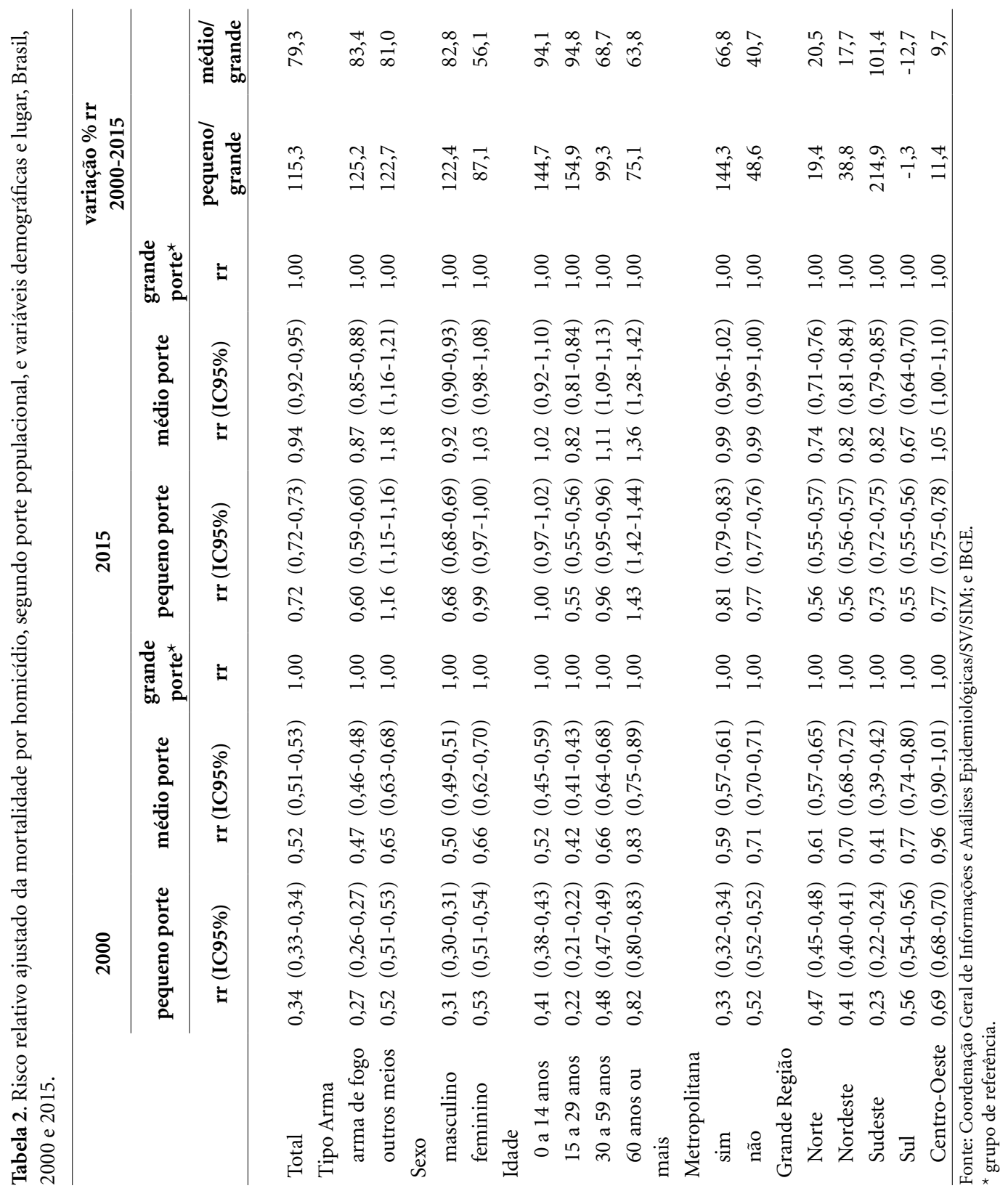

nas três categorias de porte populacional do município. Destacam-se as unidades da região Nordeste (à exceção de Pernambuco) que apresentaram os maiores incrementos das TMH do período, em particular nos municípios grandes e médios. Nos estados do Rio Grande do Norte, Sergipe, Alagoas e Paraíba os incrementos das TMH entre 2000 e 2015 foram mais acentuados em municípios de porte médio, respectivamente, $55,0,47,0$, 39,4 e 28,7 homicídios por $100 \mathrm{mil} \mathrm{ha-}$ bitantes (Figura 1).

\section{Discussão}

O risco de morte aumentou sobremaneira e mais intensamente em municípios de pequeno e médio porte no período do estudo, embora ainda apresentem as menores TMH. Exceto Sudeste e áreas não metropolitanas, a taxa também cresceu nos municípios de grande porte. $\mathrm{O}$ aumento de homicídio em todos os portes populacionais foi majoritariamente em estados das regiões Nordeste e Sul, enquanto o risco reduziu para as três 


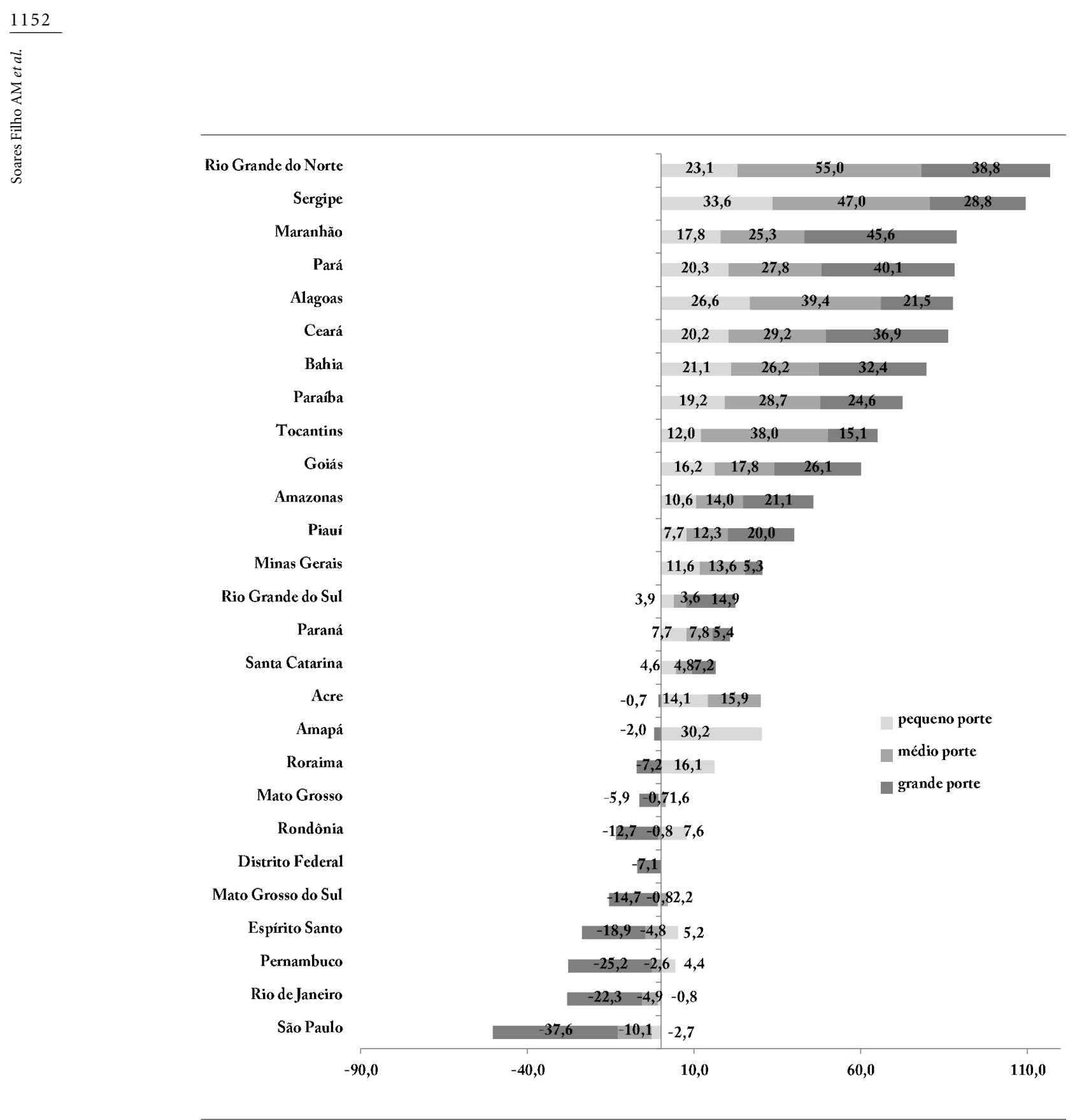

Figura 1. Variação de homicídios por 100 mil habitantes entre 2000 e 2015, segundo porte populacional e unidade federada, Brasil (excluído impacto do crescimento populacional).

Fonte: Coordenação Geral de Informações e Análises Epidemiológicas/SV/SIM; e IBGE.

categorias de município somente em estados do Sudeste.

O Brasil apresenta maiores TMH em municípios de grande porte, particularmente de áreas metropolitanas, associadas ao uso de arma de fogo, e tendo como vítimas principalmente jovens do sexo masculino. Em 2015, essas taxas foram maiores nas regiões Centro Oeste, Nordeste e Norte. Esta situação tem sido descrita por di- ferentes autores ${ }^{14,23}$. Agressão de homens jovens é associada à taxa de urbanização, desigualdades de renda, taxa de alfabetização, ocupação informal e desemprego no Brasi ${ }^{23-25}$. Tem sido descrito um círculo vicioso que se inicia na adolescência, envolvendo a ausência dos pais, abandono escolar, participação em atos infracionais menores, consumo precoce de álcool e drogas, dívidas com compras de entorpecentes, vingança e conflitos 
maiores $^{12,26-28}$. Essa exposição precoce à violência é agravada com a dependência de drogas e álcool na idade adulta ${ }^{29,30}$.

Além dos fatores sociais, violência estrutural, consumo de álcool e drogas, a violência contra mulheres aparece relacionada a histórico de agressão física e/ou sexual. Determinantes culturais exercendo função importante, observada a violência conjugal que tem o rosto de mulheres como local elegido por seus agressores. A violência doméstica avança num ambiente de rede de proteção deficiente e taxas elevadas de homicídios de grupos vulneráveis formados não somente por mulheres, em variadas cidades do país, sejam capitais ou do interior ${ }^{8,9,31,32}$.

Estados das regiões Nordeste e Norte foram os que mais contribuíram para o aumento do homicídio em municípios de diferentes portes populacionais. Taxas de homicídio de grande magnitude nos estados destas regiões coincidem com intensa e desordenada urbanização em suas capitais, marcadas por crescimento econômico e desigualdades ${ }^{15,25,33-36}$. Fatores socioeconômicos são relacionados à violência em diferentes localidades do país. O homicídio é o resultante de desigualdades sociais, em áreas caracterizadas principalmente pela miséria e pobreza, que envolve a combinação de vulnerabilidades, precariedades e rupturas de vínculos na dimensão individual e social $^{3,23,25,27,28}$.

Embora este seja o perfil da violência no Brasil já amplamente analisado, neste estudo, a observação das tendências das TMH no período de 2000 a 2015 revela um cenário ainda pouco debatido. No período de estudo (2000-2015), a tendência ascendente da TMH no Brasil ocorreu principalmente às custas do crescimento dessas taxas mais intensamente em municípios de pequeno e de médio portes, e mais discretamente nos municípios de grande porte em regiões não metropolitanas de todas as cinco regiões do Brasil. Por outro lado, o decréscimo das TMH observado no agregado dos municípios de grande porte brasileiros, é reflexo desse resultado especialmente na região Sudeste. Diferente de todas as demais regiões do Brasil, é na região Sudeste (especialmente em São Paulo, Rio de Janeiro e Espirito Santo) onde decréscimo relevante das TMH foi observado entre 2000 e 2015. Essas tendências, em geral, fizeram com que o risco de morte por homicídios entre municípios de pequeno e médio portes se aproximassem ao risco dos municípios de grande porte no Brasil.

A redução da violência observada em determinadas localidades possivelmente repercute de modo distinto nos diferentes grupos sociais, a exemplo da diminuição do risco de homicídio na população branca e a discreta afetação das taxas na população negra no Brasil ${ }^{6}$. Políticas públicas gerais podem não atender a necessidades específicas de indivíduos que demandam medidas particulares.

A mudança de padrão de mortalidade por homicídio observada neste estudo indica certa recomposição da violência no espaço nacional, caracterizada por ampliação e certo grau de interiorização desses eventos ${ }^{14,23,33-36}$. A expansão e a integração de zonas urbanas aproximam variados tipos de cidades e aglomerados regionais e permitem algum efeito de contágio, o que pode fazer parte da explicação do fenômeno de interiorização da violência identificado nesse estudo $^{36-38}$. Além disso, a integração do interior do Brasil à dinâmica da economia nacional, desconcentração industrial e mobilidade da fronteira agrícola são fatores apontados como responsáveis pela dispersão espacial da população e sua maior distribuição na rede urbana nas últimas décadas ${ }^{38}$. Esse movimento de maior urbanização da população brasileira converte cidades médias e arredores de áreas perimetropolitanas em polos de atração de migrações internas e inter-regionais, já que oferecem equipamentos, serviços e empregos que podem atender, em certa medida, as demandas regionais. Assim, se identifica uma dinâmica de influência socioeconômica e funcional de cidades-sede sobre cidades menores ${ }^{37}$. Assim, o homicídio e outros crimes se deslocam e avançam em direção a municípios do interior do Brasil $^{33,36,39,40}$. Esse conjunto de mudanças pode estar acompanhado por condições propícias ao crescimento da morte violenta e outros crimes em áreas não metropolitanas. Aliado a isso, vale lembrar que a maioria dos serviços públicos, a exemplo de aparato policial, se concentra nas grandes cidades, em especial nas localizadas em regiões metropolitanas, reprimindo fortemente a violência nestas regiões e também forçando seu deslocamento em direção ao interior do país ${ }^{39}$.

$\mathrm{O}$ aumento das TMH em agregados de municípios de todos os portes populacionais foi observado, neste estudo, nas regiões Norte, Nordeste, Centro-Oeste e Sul. Padrão distinto foi observado, no entanto, na região Sudeste, onde o risco de homicídio aumentou nos municípios de pequeno porte e reduziu nos de grande porte. A redução da morte violenta nos grandes municípios da região Sudeste pode estar relacionada à mudança demográfica, melhoria e equidade de renda, aumento das oportunidades escolares e políticas 
de segurança, como desarmamento e encarceramento $^{41}$. Na cidade de São Paulo, por exemplo, o decréscimo do homicídio ocorreu em praticamente todos os grupos populacionais, mas com maior intensidade entre homens, jovens, de áreas de maior vulnerabilidade social ${ }^{16,42,43}$. Evidências apontam para a maior importância dos fatores socioeconômicos e principalmente alterações na estrutura etária, com alguma atribuição da implantação de polícia comunitária em favelas e de certos dispositivos extralegais de regulação interna ao "mundo do crime" que se legitimam nas periferias, de maneira semelhante ao observado na cidade do Rio de Janeiro ${ }^{41,44-47}$.

O presente estudo foi completamente desenvolvido com uso de dados secundários de mortalidade e demográficos. Este fato encerra limitações que merecem atenção. Por exemplo, a existência de códigos inespecíficos, tais como eventos de intenção indeterminada, nos dados de mortalidade por causas externas, pode acarretar limitações desta análise ${ }^{48}$. No intuito de amenizar a repercussão da exclusão desses casos, optou-se, conforme descrito nos métodos, por considerar alguns desses códigos (Y22-Y24) como homicídio. Ainda assim, certo erro de classificação pode ter ocorrido. Além disso, o uso de apenas dois anos na análise das tendências das TMH pode omitir variações relevantes intraperíodos. Estudos futuros podem ter esse objetivo.

Vale lembrar ainda que fatores que atuaram para a redução do homicídio, principalmente em grandes cidades do Sudeste, observada neste estudo até 2015, exibiram mudanças de percurso consideráveis nos últimos anos. Vigorosa crise econômica e das finanças públicas de 2014 a 2017, acompanhada de perda da capacidade financeira do governo e ampliação do desemprego, criaram potencial para terem afetado mais intensamente determinadas unidades federadas. Isso pode ter ocorrido, por exemplo, com o Rio de Janeiro, e ter levado a perdas e ao recrudescimento de conquistas anteriores ${ }^{49}$. Essa questão merece ser continuamente estudada. Políticas públicas sociais e de segurança tornam-se cada vez mais frágeis. Tal situação pode reverter a tendência de redução no Sudeste e exacerbar a intensidade do risco de homicídio nas regiões Norte e Nordeste. Finalmente, investigações futuras são necessárias para demonstrar o grau de relação de aumento do homicídio com municípios de pequeno porte e determinar claramente a sua etiologia. Estas são importantes para a priorização de políticas que identifiquem as características de aglomerados de alta e baixa prioridade, bem como o apoio a boas práticas de políticas públicas bem sucedidas que possam ser reproduzidas em outras localidades onde a violência apresenta tendência ascendente.

A violência urbana é sabidamente multicau$\mathrm{sal}^{28,30,50-52}$. Ela se fundamenta na escassez e/ou ineficiência da presença do Estado, com precariedade de políticas sociais e de segurança pública e escasso acesso à justiça. Ela é potencializada pelas desigualdades sociais, somada à alta proporção de jovens, altas taxas de abandono escolar, analfabetismo e desemprego, acesso e consumo de álcool e drogas, disponibilidade de armas, conflitos de grupos, violência doméstica e desagregação familiar. É óbvio, portanto, o entendimento de que o enfrentamento dessa violência urbana exige ações intrassetoriais, coordenadas e continuadas, dirigidas às suas mais diversas etiologias e não apenas às suas consequências mais superficiais. A produção de evidências procedentes de investigações, que elucidem as razões das tendências observadas neste estudo, pode auxiliar sobremaneira a maior efetividade das ações, em particular quanto ao crescimento da violência em municípios de pequeno e médio porte brasileiros. Como expressão mais explícita da violência e, dada a sua magnitude, o homicídio consiste em sério problema social com intensas repercussões para o setor saúde, devendo ser objeto recorrente de análise e de políticas públicas.

\section{Colaboradores}

AM Soares Filho contribuiu na concepção, desenho do estudo, aquisição dos dados e, análise e interpretação dos dados. AM Soares Filho, EC Duarte e E Merchan-Hamann contribuíram na redação das versões preliminares, aprovaram a versão final do artigo e declaram-se responsáveis por todos os aspectos do trabalho, no sentido de garantir que as questões relacionadas à exatidão ou à integridade de qualquer parte da obra sejam devidamente investigadas e resolvidas. 


\section{Referências}

1. Institute for Health Metrics and Evaluation. Data Visualization. c2015. [database on the Internet]. Seattle: 2017. [acessado 2017 Ago 10]. Disponível em: http:// ghdx.healthdata.org/gbd-results-tool

2. Ribeiro M, Dunn J, Laranjeira R, Senso R. High mortality among young crack cocaine users in Brazil: a 5-year followup study. Adicction 2004; 99 (9):11331135.

3. Tavares R, Catalan VDB, Romano MM, Melo EM. Homicídios e vulnerabilidade social. Cien Saude Colet 2016; 21 (3):923-934.

4. Cruz JM. La victimización por violência urbana: niveles e factores associados en ciudades de América Latina y Espana. Rev Panam de Salud Publica 1999; 5(4/5):259-267.

5. Andreuccetti G, Carvalho HB, Carvalho Ponce J, Carvalho DG, Kahn T, Muñoz DR, Leyton V. Alcohol consumption in homicide victims in the city of São Paulo. Addiction 2009; 104(12):1998-2006.

6. Soares Filho AM. Vitimização por homicídios segundo características de raça no Brasil. Rev Saude Publica 2011; 45(4):745-755.

7. Silva LS, Meneses MLN, Lopes CLA, Correa MSM Anos potenciais de vida perdidos por mulheres vítimas de homicídio na cidade do Recife, Pernambuco, Brasil. Cad Saude Publica 2011; 27(9):1721-1730.

8. Amaral NA, Amaral CA, Amaral TLM. Mortalidade feminina e anos de vida perdidos por homicídio/ agressão em capital brasileira após promulgação da lei Maria da Penha. Texto Contexto Enferm 2013; 22(4):980-988

9. Silva MA, Cabral Filho JE, Amorim MMR, Falbo Neto GH. Mulheres vítimas de homicídio em Recife, Pernambuco, Brasil, 2009/2010: um estudo descritivo. Cad Saude Publica 2013; 29(2):391-396.

10. Bastos MJRP, Pereira JA, Smarzaro DC, Costa EF, Bossanel RCL, Oliosa DMS, Pereira JMP, Feitosa HN, Costa MF, Oliveira FJP, Fávero JL, Maciel ELN. Análise ecológica dos acidentes e da violência letal em Vitória, ES. Rev Saude Publica 2009; 43(1):123-132.

11. Cardona M, García HI, Giraldo CA, López MV, Suárez CM, Corcho DC, Posada CH, Flórez MN. Homicidios en Medellín, Colombia, entre 1990 y 2002: actores, móviles y circunstancias. Cad Saude Publica 2005; 21(3):840-851.

12. Kodato S, Silva APS. Homicídios de adolescentes: refletindo sobre alguns fatores associados. Psicol. Reflex. Crit. 2000; 13(3):507-515.

13. Nachif MCA. Homicide as a public health problem in the city of Campo Grande, Mato Grosso do Sul, Brazil. Psicol. Soc. 2006; 18(2):99-104.

14. Malta DC, Minayo MCS, Soares Filho AM, Silva MMA, Montenegro MMS, Ladeira RM, Morais Neto OL, Melo AP, Mooney M, Naghavi M. Mortalidade e anos de vida perdidos por violências interpessoais e autoprovocadas no Brasil e Estados: análise das estimativas do Estudo Carga Global de Doença, 1990 e 2015. Rev. Bras. Epidemiol. 2017; 20(Supl. 1):142-156.

15. Mendes LVP, Campos MR, Von-Doellinger VR, Mota JC, Pimentel TG, Schramm JMA. A evolução da carga de causas externas no Brasil: uma comparação entre os anos de 1998 e 2008. Cad Saude Publica 2015; 31(10):2169-2184.
16. Andrade LT, Diniz AMA. A reorganização espacial dos homicídios no Brasil e a tese da interiorização. $R$. Bras. Est. Pop. 2013; 30(Supl.):S171-S191.

17. Brasil. Ministério da Saúde (MS). Informações de saúde: estatísticas vitais. [base de dados na Internet]. Brasília: MS; 2017. [acessado 2017 Jul 12]. Disponível em: http://tabnet.datasus.gov.br/cgi/deftohtm.exe?sim/cnv/ext10br.def

18. Brasil. Ministério da Saúde (MS). Informaçães de saúde: demográficas e socioeconômicas. [base de dados na Internet]. Brasília: MS; 2017. [acessado 2017 Jul 12]. Disponível em: http://tabnet.datasus.gov.br/cgi/ deftohtm.exe?novapop/cnv/popbr.def

19. Organização Mundial da Saúde (OMS). Classificação Estatística Internacional de Doenças e Problemas Relacionados à Saúde. São Paulo: Ed. USP; 1993.

20. Castro MSM, Assunção RM, Durante MO. Comparação de dados sobre homicídios entre dois sistemas de informação, Minas Gerais. Rev Saude Publica 2003; 37(2):168-176

21. Brasil. Decreto $n^{\circ} 7.724$, de 16 de maio de 2012. Regulamenta a Lei no 12.527 , de 18 de novembro de 2011 que dispõe sobre o acesso a informações. Diário Oficial da União 2012; 18 maio.

22. Brasil. Ministério da Saúde (MS). Resolução $\mathrm{n}^{\circ}$ 510, de 7 de abril de 2016. Dispõe sobre as normas aplicáveis a pesquisas em Ciências Humanas e Sociais. Diário Oficial da União 2016; 24 maio.

23. Duarte EC, Garcia LP, Freitas LRS, Mansano NH, Monteiro RA, Ramalho WM. Associação ecológica entre características dos municípios e o risco de homicídios em homens adultos de 20-39 anos de idade no Brasil, 1999-2010. Cien Saude Colet 2012; 17(9):2259-2268.

24. Andrade L, Nihei OK, Pelloso SM, Carvalho MDB. Homicídios juvenis e informalidade em um município brasileiro da tríplice fronteira Brasil, Paraguai e Argentina. Rev Panam Salud Publica 2012; 31(5):380-387.

25. Bando DH, Lester D. Estudo ecológico sobre suicídio e homicídio no Brasil. Cien Saude Colet 2014; 19(4):1179-1189.

26. Jimenez L, Frasseto FA. Face da morte: a lei em conflito com o adolescente. Psicol Soc 2015; 27(2):404-414.

27. Sousa GS, Magalhães FB, Gama IS, Lima MVN, Almeida RLF, Vieira LJES, Bezerra Filho JG. Determinantes sociais e sua interferência nas taxas de homicídio em uma metrópole do nordeste brasileiro. Rev Bras Epidemiol 2014; 17(Supl. DSS):194-203.

28. Caicedo B, Gonçalves H, González DA, Victora CG. Violent delinquency in a Brazilian birth cohort: the roles of breast feeding, early poverty and demographic factors. Paediatr Perinat Epidemiol 2010; 24(1):1223.

29. Madruga CS, Laranjeira R, Caetano R, Ribeiro W, Zaleski M, Pinsky I, Ferri CP. Early life exposure to violence and substance misuse in adulthood: the first Brazilian national survey. Addictive Behaviors 2011; 36(3):251-255

30. Gorman DM, Ponicki WR, Zheng Q, Gruenewald PJ, Gaidus AJ. Violent crime redistribution in a city following a substantial increase in the number of off-sale alcohol outlets: a Bayesian analysis. Drug Alcohol Rev 2018; 37(3):348-355 
31. Leites GT, Meneghel SN, Hirakata VN. Homicídios femininos no Rio Grande do Sul, Brasil. Rev Bras Epidemiol 2014; 17(3):642-653.

32. Lamoglia CVA, Minayo MCS. Violência conjugal, um problema social e de saúde pública: estudo em uma delegacia do interior do Estado do Rio de Janeiro. Cien Saude Colet 2009; 14(2):595-604.

33. Lima MLC, Ximenes RA, Feitosa CL, Souza ER, Albuquerque MFPM, Barros MDA, Souza WV, Lapa TM. Conglomerados de violência em Pernambuco, Brasil. Rev Panam Salud Publica 2005; 18(2):122-128.

34. Souza ER, Lima MLC. The panorama of urban violence in Brazil and its capitals. Cien Saude Colet 2006; 11(2):363-373.

35. Lozada EMK, Mathias TAF, Andrade SM, Aidar T. Tendência da mortalidade por homicídios no estado do Paraná, segundo Regionais de Saúde, 1979 a 2005. Rev Bras Epidemiol 2009; 12(2):258-269.

36. Souza TO, Pinto LW, Souza ER. Estudo espacial da mortalidade por homicídio, Bahia, 1996-2010. Rev Saude Publica 2014; 48(3):468-477.

37. Gurgel APC. As metrópoles do interior do Nordeste: a caracterização de um tipo metropolitano regional. Cadernos Metrópole 2017; 19(40):841-864.

38. Stamm C, Staduto JAR, Lima JF, Wadi YM. A população urbana e a difusão das cidades de porte médio no Brasil. Interações 2013; 14(2):251-265.

39. Scorzafave LG, Justus M, Shikida PFA. Safety in the global south: criminal victimization in Brazilian rural areas. Journal of Rural Studies 2015; 39:247-261.

40. Andrade SM, Soares DA, Souza RKT, Matsuo T, Souza HD. Homicídios de homens de quinze a 29 anos e fatores relacionados no estado do Paraná, de 2002 a 2004. Cien Saude Colet 2011; 16(Supl. 1):1281-1288.

41. Mello JMP, Schneider A. Mudança demográfica e a dinâmica dos homicídios no estado de São Paulo. São Paulo em Perspectiva 2007; 21(1):19-30.

42. Peres MFT, Almeida JF, Vicentin D, Cerda M, Cardia N, Adorno S. Queda dos homicídios no Município de São Paulo: uma análise exploratória de possíveis condicionantes. Rev Bras Epidemiol 2011; 14(4):709721.

43. Nadanovsky P. O aumento no encarceramento e a redução nos homicídios em São Paulo, Brasil, entre 1996 e 2005. Cad Saude Publica 2009; 25(8):18591864.
44. Peres MFT, Almeida JF, Vicentin D, Ruotti C, Nery MB, Cerda M, Cardia N, Adorno S. Evolução dos homicídios e indicadores de segurança pública no Município de São Paulo entre 1996 a 2008: um estudo ecológico de séries temporais. Cien Saude Colet 2012; 17(12):3249-3257.

45. Feltran GS. Crime e castigo na cidade: os repertórios da justiça e a questão do homicídio nas periferias de São Paulo. Caderno CRH 2010; 23(58):59-73.

46. Barcellos C, Zaluar A. Homicídios e disputas territoriais nas favelas do Rio de Janeiro. Rev Saude Publica 2014; 48(1):94-102.

47. Cardoso FLMG, Cecchetto FR, Corrêa JS, Souza TO. Homicídios no Rio de Janeiro, Brasil: uma análise da violência letal. Cien Saude Colet 2016; 21(4):12771288.

48. Soares Filho AM, Cortez-Escalante JJ, França E. Revisão dos métodos de correção de óbitos e dimensões de qualidade da causa básica por acidentes e violências no Brasil. Cien Saude Colet 2016; 21(12):3803-3818.

49. Barbosa Filho FH. A crise econômica de 2014/2017. Estud Av 2017; 31(89):51-60.

50. Drumond EF, Souza HNF, Hang-Costa TA. Homicídios, álcool e drogas em Belo Horizonte, Minas Gerais, Brasil, 2000-2009. Epidemiol Serv Saúde 2015; 24(4):607-616.

51. Peres MFT, Santos PC. Mortalidade por homicídios no Brasil na década de 90: o papel das armas de fogo. Rev Saude Publica 2005; 39(1):58-66.

52. Murray J, Cerqueira DRC, Kahn T. Crime and violence in Brazil: Systematic review of time trends, prevalence rates and risk factors. Aggression and Violent Behavior 2013; 18(5):471-483.

Artigo apresentado em 27/04/2018

Aprovado em 19/07/2018

Versão final apresentada em 21/07/2018 